\title{
High-speed multichannel impedance measuring system
}

\author{
Marco J. da Silva, Eduardo N. dos Santos, Tiago P. Vendruscolo \\ Department of Electrical Engineering, Universidade Tecnológica Federal do Paraná, Av. Sete de Setembro 3165, 80230-901 Curitiba-PR, \\ Brazil
}

In this paper, a novel high-speed multichannel impedance measuring system in presented. The measurements are based on simultaneous excitation with two distinct frequencies to interrogate the multiple sensing point of a given sensor. Received signals are analogue-to-digital converted (with a DAQ card) and the amplitudes of each frequency are determined using FFT implemented in LabVIEW. The capacitive and conductive parts of impedance are calculated based on amplitude measurements. The developed system can operate 8 transmitter and 8 receiver electrodes at a frame repetition frequency of up to $781 \mathrm{~Hz}$, i.e. single channels are sampled at $6,248 \mathrm{~Hz}$. The system has been evaluated by measuring reference components. Deviations from references values are below $10 \%$ which considering the fast repetition frequency of measurements is satisfactory. The developed system was applied to visualize the fluid distribution over the surface of planar multipoint sensor. Two different liquids (oil and water) and air were evaluated and their spatial distribution over the sensor's surface was correctly visualized.

Keywords: Electrical impedance; multichannel; high-speed measurement; fluid distribution measurement; planar sensor

Citation: Marco J. da Silva, Eduardo N. dos Santos, Tiago P. Vendruscolo, High-speed multichannel impedance measuring system, Acta IMEKO, vol. 1, no. 1, article 9, July 2012, identifier: IMEKO-ACTA-01(2012)-01-09

Editor: Pedro Ramos, Instituto de Telecomunicações and Instituto Superior Técnico/Universidade Técnica de Lisboa, Portugal

Received January 24 $4^{\text {th }}, 2012$; In final form June $1^{\text {st }}, 2012$; Published July 2012

Copyright: (c) 2012 IMEKO. This is an open-access article distributed under the terms of the Creative Commons Attribution 3.0 License, which permits unrestricted use, distribution, and reproduction in any medium, provided the original author and source are credited

Funding: This work was partially funded by BG Group, Brazil and Petrobras, Brazil

Corresponding author: Marco J. da Silva, e-mail: mdasilva@utfpr.edu.br

\section{INTRODUCTION}

Impedance sensors, in which the measurand causes a variation of an electrical characteristics such as resistance or capacitance, have found widespread use in industrial applications mainly due to their simplicity, low fabrication costs and robustness [1,2]. Impedance measurement is a common tool for the characterization of electrical properties of materials and substances, in which measurement times of seconds to minutes are used to achieve high measurement accuracy in the chemical analysis [3,4]. However, in industrial applications, measuring times in the range of microseconds to milliseconds are required in order to investigate dynamic processes, e.g. mixing of substances in chemical reactors, or multiphase flow in pipelines [5]. Accuracy requirements in such applications are less critical, since substances involved, and consequently their electrical properties, are known a-priori. In addition, multipoint impedance measurement is also often required in order to obtain spatial or distributed information, for instance, in the imaging of multiphase flows [6,7] or for the measurement of distributed sensors [8-14].

Hitherto used systems for multichannel measurement are limited to the evaluation of a single electric parameter such as resistance or capacitance [7-14]. Commercial measuring instruments such as LCR meters can only reach repetition rates of few measurements per second and are, therefore, not suitable for high-speed measurements which are necessary in some industrial application, such as the investigation of fluid flow.

In recent years some efforts have been made by applying dual-modality measuring techniques for enhancing the range of application in which two different sensing techniques are used to distinguish more than two substances. Furthermore, the use of single-point electrical impedance/admittance measurements, i.e. the measurement of both real and imaginary parts (or amplitude and phase), has been reported in the past [15-18].

In this paper, we introduce a novel high-speed multichannel electronics being able to simultaneously determine the conductive and the capacitive component of a multipoint sensor which is arranged in a matrix-like form such as wire-mesh sensors [6,7] or planar array sensors [12-14]. Circuits are based on classical impedance measurement techniques and the basic idea of two components evaluation is to apply an excitation signal composed of two distinct frequencies. Based on amplitude measurements only and spectral analysis of data it is possible to determine the resistive and capacitive components at each sensing point. Multichannel interrogation is achieved by a multiplexed excitation-sensing scheme. 
In section 2 the basic measuring principle is first described and then the multichannel electronics is presented. The new electronics is evaluated in section 3. An application example is given in section 4, in which a planar array sensor is used to visualize the spatial distribution of different fluids over the sensor surface. The main achievements are summarized in the end.

\section{SYSTEM DEVELOPMENT}

\subsection{One-channel circuit analysis}

For impedance measurements we chose the circuit synonymously known as transimpedance amplifier, autobalancing bridge or current-voltage converter, as depicted in Figure 1. Since the developed system is meant to be used to identify different fluids in an approach similar to [13], the electric model that better represents the characteristics of a fluid is a parallel RC circuit $[19,20]$. Thus, the unknown impedance was assumed to be capacitive only. In Figure $1, V_{\mathrm{i}}$ is the excitation voltage, $Z_{\mathrm{x}}$ represents the unknown impedance and $C_{\mathrm{f}}$ along with $R_{\mathrm{f}}$ the feedback network. Furthermore, $C_{\mathrm{s} 1}$ and $C_{\mathrm{s} 2}$ represent the stray capacitances to ground which are caused, for instance, by cables used to connect the circuit with a sensor. In principle these stray capacitances have no influence in the circuit since $C_{\mathrm{s} 1}$ is directly driven by the source voltage and $C_{\mathrm{s} 2}$ is virtually grounded by the opamp.

The impedance is determined by measuring the voltage at the opamp output. Assuming that the opamp is ideal the complex-value output voltage $\mathbf{V}_{\text {o }}$ is determined by

$$
\frac{\mathbf{V}_{\mathrm{o}}}{\mathbf{V}_{\mathrm{i}}}=-\left(\frac{G_{\mathrm{x}}+j \omega C_{\mathrm{x}}}{G_{\mathrm{f}}+j \omega C_{\mathrm{f}}}\right),
$$

where $\omega=2 \pi f, f$ is the frequency of the sinusoidal excitation signal and $j$ is the imaginary unit $\left(j^{2}=-1\right)$.

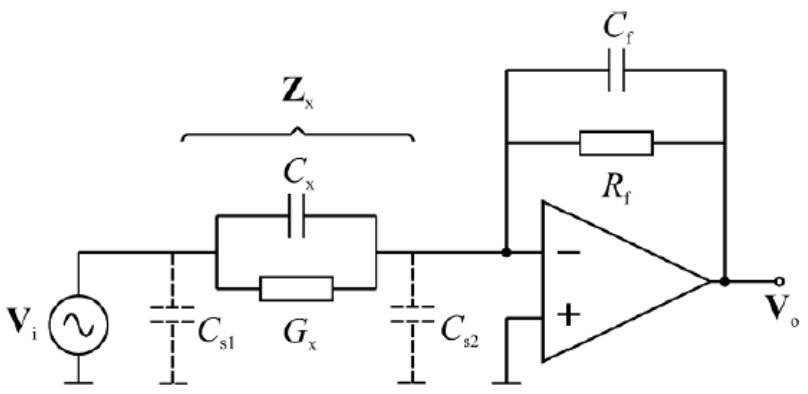

Figure 1. Practical circuit for measuring impedances formed as parallel circuit of a capacitor and a resistor.

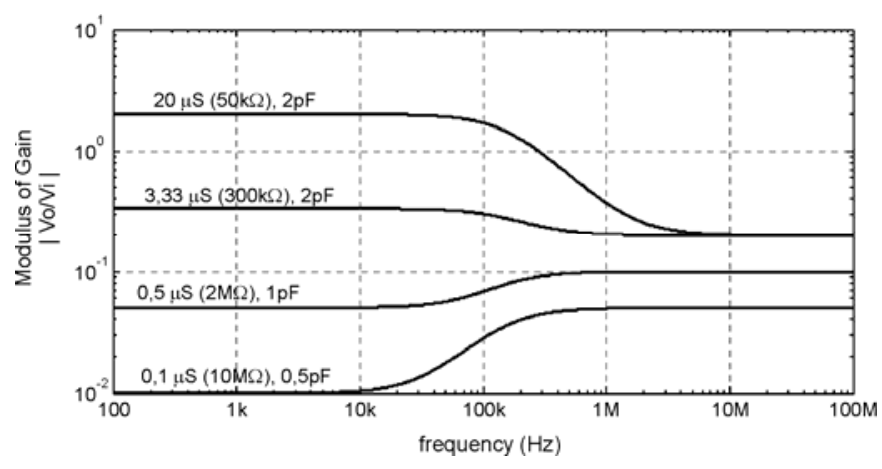

Figure 2. Frequency response for components values $C_{\mathrm{f}}=10 \mathrm{pF}, G_{\mathrm{f}}=10 \mu \mathrm{S}$ $(100 \mathrm{k} \Omega), C_{\mathrm{x}}$ and $G_{\mathrm{x}}\left(R_{\mathrm{x}}\right)$ are indicated in the plots.
The Bode Diagram for the amplitude of eq. (1), using typical $\mathrm{R}$ and $C$ values, is shown in Figure 2. Two plateaus can be readily identified - one at low frequencies and the other at high frequencies. Knowing that a capacitor works as an open circuit in DC, the smaller the frequency, the lower the influence of the capacitive part, that is, the impedance is purely resistive. On the other hand, the higher the frequency, the higher the influence of the capacitive part and the lower is the resistive part relative contribution.

The magnitude of each plateau is given by the quotient of $G_{\mathrm{x}} / G_{\mathrm{f}}$ and $C_{\mathrm{x}} / C_{\mathrm{f}}$ which are obtained taking the limit for $f \rightarrow 0$ and $f \rightarrow \infty$ of the modulus of equation (1), in the form

$\left|\frac{\mathbf{V}_{\mathrm{o}}}{\mathbf{V}_{\mathrm{i}}}\right|=\frac{\sqrt{G_{\mathrm{x}}^{2}+(2 \pi f)^{2} C_{\mathrm{x}}^{2}}}{\sqrt{G_{\mathrm{f}}^{2}+(2 \pi f)^{2} C_{\mathrm{f}}^{2}}}$.

A simplified way to view the behaviour of an auto-balancing bridge is according to the graph showing in the Figure 3. Further shown in the figure, is the operational amplifier frequency response.

In principle any two frequencies may be chosen for determining the unknown components. The simplest choice, however, is to select two frequencies located exactly in each plateau. Hence, the two unknown parameters are found by

$G_{\mathrm{x}}=G_{f}\left|\frac{\mathbf{V}_{\mathrm{o}}}{\mathbf{V}_{\mathrm{i}}}\right|_{f=f_{\text {low }}}$

and

$C_{\mathrm{x}}=C_{f}\left|\frac{\mathbf{v}_{\mathrm{o}}}{\mathbf{V}_{\mathrm{i}}}\right|_{f=f_{\text {high }}}$.

Since the resistance $R$ is directly proportional to the conductivity of the material, and the capacitance $C$ is directly proportional to the electric permittivity of the material, the measurement of the resistance and capacitance is an indication of the electrical conductivity and permittivity of the substance in question. Substances can thus be distinguished from each other and thus correctly indentified based on these measurements.

\subsection{Multichannel system}

The developed multichannel system is schematically shown in Figure 4. The developed hardware is basically divided into four blocks. The first is the transmitter printed-circuit board (PCB), in which there are two direct digital synthesizers (AD9833 from Analog Devices) for generating the two

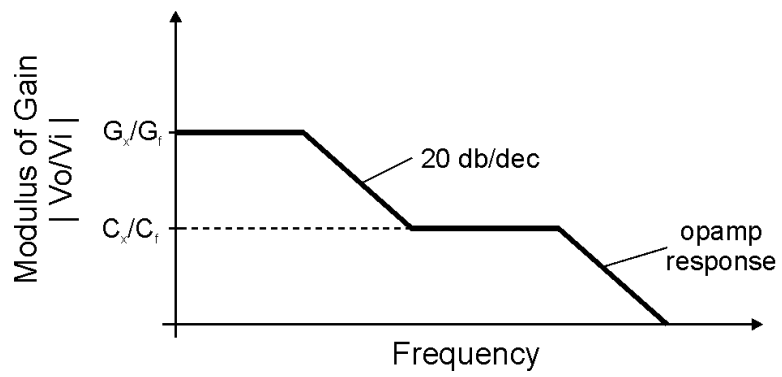

Figure 3. Asymptotic and simplified frequency response of a practical circuit for impedance measurement considering the opamp non-ideal frequency response. 


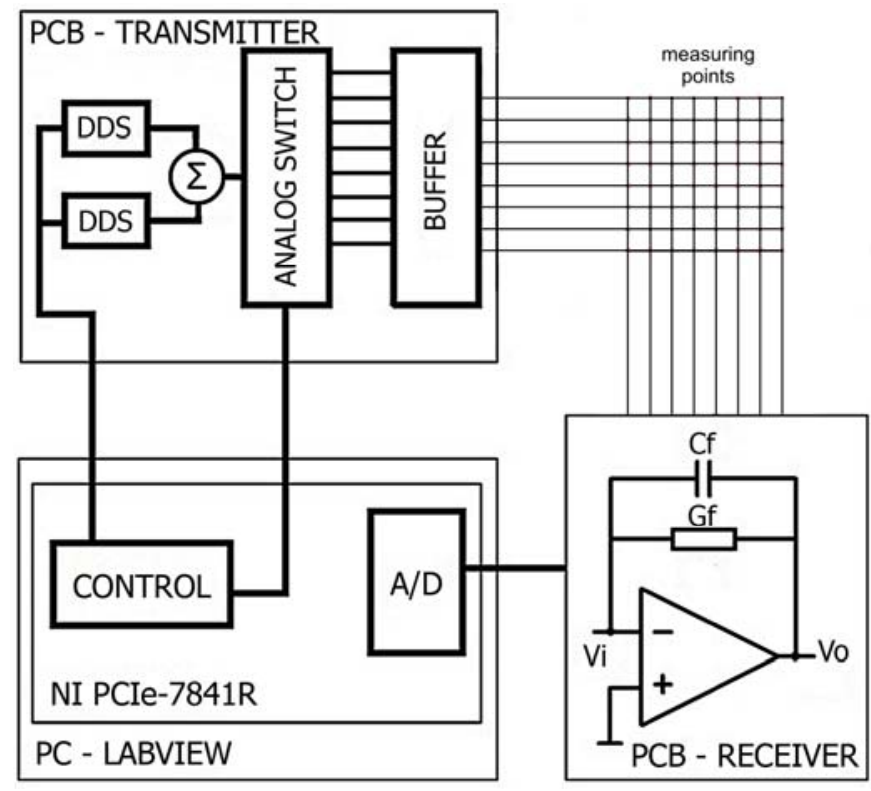

Figure 4. Block diagram of the developed multichannel impedance measuring system.

sinusoidal signals at different frequencies. The direct digital synthesizer (DDS) is a programmable integrated circuit, which can generate signals up to $12.5 \mathrm{MHz}$. The DDS are programmed via the employed data acquisition card (PCIe-7841R from National Instruments) using a Serial Peripheral Interface (SPI) and are user-defined via software developed in LabVIEW.

As described in the previous section, two frequencies are sufficient for the evaluation of parameters $R$ and $C$. In this way, a signal composed by two single frequencies is generated by summing together the signals of two separated DDS circuits by means of an adder circuit using operational amplifiers. The excitation signal (containing two frequencies) is connected to up to eight excitation electrodes with the help of analogue switches (ADG1434). The non-selected channels are grounded in order to allow for a multiplexed excitation scheme. Buffers (LMH6722) at the output assure that the excitation signals have low impedance. The signal is than connected to the transmitter electrodes of a given sensor. The receiver electrodes of the sensor are connected to the receiver board, where transimpedance amplifiers (Figure 2) convert the signals into proportional voltage, as described in section 2.1 , whereby the operational amplifier used is the OPA656 $(500 \mathrm{MHz}$ unity gain bandwidth) with feedback components of $4.7 \mathrm{pF}$ and $100 \mathrm{k} \Omega$. The voltage signals from the opamp are A/D-converted by the acquisition card. The digitalized signals are processed in the

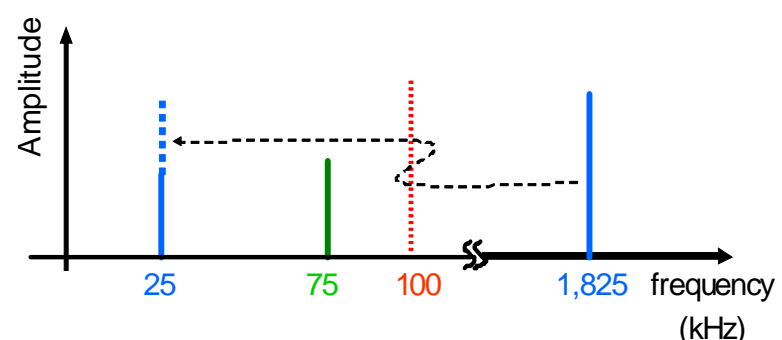

Figure 5. Schematic representation of amplitude spectrum obtained via FFT analysis. host PC with a program created in LabVIEW. The amplitudes of the excitation signals are determined using Fast Fourier Transform (FFT) processing tools. In order to allow for fast response, each channel is sampled at $200 \mathrm{kHz}$ (maximum possible sampling frequency - simultaneous sampling) and 32 samples are processed. For the reason that for one single image to be obtained, 8 transmitter electrodes must be activated, up to $781 \mathrm{~Hz}$ frame rate is possible for sensors arranged in an $8 \times 8$ matrix configuration.

Although the PCIe-7841R analogue input channels have a bandwidth $(-3 \mathrm{~dB})$ of $1 \mathrm{MHz}$, high frequency sinusoidal signal was configured to $1.825 \mathrm{MHz}$ in order to reach the capacitance plateau as schematically shown in Figure 3. The voltage signal at $1.825 \mathrm{MHz}$ is attenuated by $-6.45 \mathrm{~dB}$. The low frequency signal used was $75 \mathrm{kHz}$. Since the sampling frequency is $200 \mathrm{kHz}$ (Nyquist frequency $=100 \mathrm{kHz}$ ), the $1.825 \mathrm{MHz}$ signal is undersampled, appearing as $25 \mathrm{kHz}$ component in the FFT spectrum, as schematically shown in Figure 5. Though undersampled and attenuated, the amplitude of the high frequency component can be correctly evaluated after calibration measurements, i.e. measurements with known impedances or fluids.

\section{SYSTEM EVALUATION}

The developed system was evaluated to verify the performance of step and frequency responses. Further, accuracy of the developed system was evaluated by measuring different impedances with known values consisting of a parallel RC circuit, as described below.

\subsection{Frequency response}

A sinusoidal voltage signal swept in frequency from $2 \mathrm{kHz}$ to $20 \mathrm{MHz}$ was used to test the frequency response of the auto-balancing bridge circuit. The signal was applied to the input of a known RC circuit (measurand) which was connected to the auto-balancing bridge circuit. The output signal of the receiver module was measured with an oscilloscope. Thus, we surveyed the frequency response curves for four different combinations of resistors and capacitors with values similar to the ones in Figure 2 for comparison. The obtained frequency responses are depicted in Figure 6.

The two plateaus can be clearly seen in the experimental results. Also, the shape of the curves fits very well with the theoretical response, as anticipated in section 2.1. Only the absolute measured values (Figure 6) slightly deviate from the theoretical ones (Figure 2). This fact implies that the circuit

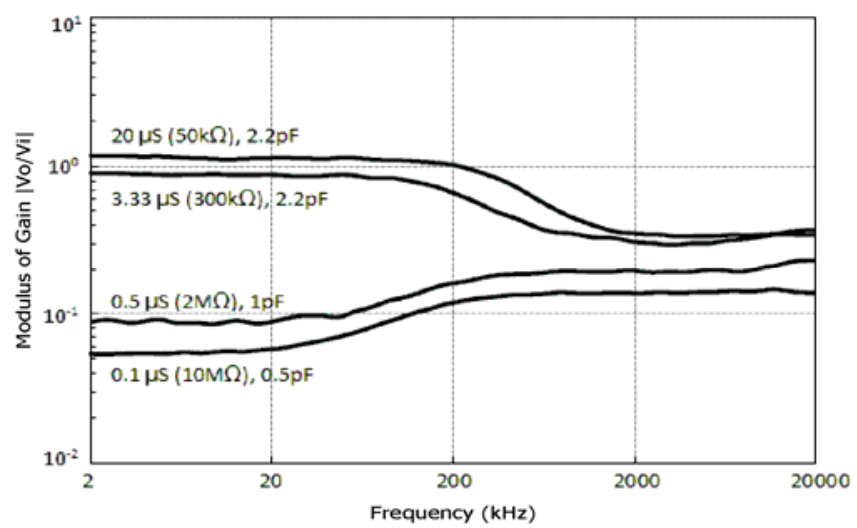

Figure 6. Frequency response in circuit for components values $C_{\mathrm{f}}=10 \mathrm{pF}$, $G_{\mathrm{f}}=10 \mu \mathrm{S}(100 \mathrm{k} \Omega), C_{\mathrm{x}}$ and $G_{\mathrm{x}}\left(R_{\mathrm{x}}\right)$ are indicated in the plots. 
input-output response must be adjusted by using some reference components.

\subsection{Step response}

In order to verify the maximum achievable repetition in a multichannel system, the step response of the auto-balancing bridge circuit was evaluated. Using a waveform generator capable of amplitude modulation (Agilent 33220A), in which a carrier sine wave of $1 \mathrm{MHz}$ was modulated with a square wave signal of $50 \mathrm{kHz}$, the step response of the circuit can be evaluated. An oscilloscope at the output shows the resulting waveform, as depicted in Figure 7. In this experiment the RC circuit used was a $2.2 \mathrm{pF}$ capacitance in parallel with a $1 \mathrm{M} \Omega$ resistance.

As shown in the Figure 7, the time response of the carrier $(1 \mathrm{MHz})$ is almost instantaneous for the time scale used, so that the system can be properly used in the intended kilohertz repetition range with no loss in measurement accuracy.

\subsection{Static impedance measurement}

In order to evaluate the accuracy of the measuring circuit, different combinations of known resistor and capacitors were measured for a single transmitter-receiver pair, which is representative for all channels. Excitation amplitude of two components was kept constant, and the amplitude of output signals was determined via 32-point FFT and at a repetition rate of $6.248 \mathrm{kHz}$ (single channel measurement), as described earlier. Twenty two combinations of resistors and capacitors were measured, with values between $100 \mathrm{k} \Omega-2.2 \mathrm{M} \Omega$ and $1 \mathrm{pF}-10 \mathrm{pF}$. The parity plots of measured (eq. 3 and 4) and reference values are shown in Figures 8 and 9. Deviations from references values are below $10 \%$ which is satisfactory considering the fast repetition frequency. Higher accuracy may be achieved by lowering the repetition frequency of measurements, thus decreasing random errors.

\section{EXAMPLE OF AN APPLICATION}

In this section, the developed electronics is used to operate a planar array sensor. The sensor is composed by a number of identical sensing elements which are individually interrogated by the electronics. As an example of application, the spatial distribution of two different liquids and of air is visualized based on their electrical properties.

\subsection{Planar array sensor}

The sensor employed in this section is composed of 256 single interdigital sensing structures which are multiplexed in a matrix with 16 driver (rows) and 16 sensing electrodes (columns). Figure 10 depicts the sensor. Since the developed electronics can handle $8 \times 8$ electrodes, only one quarter of the

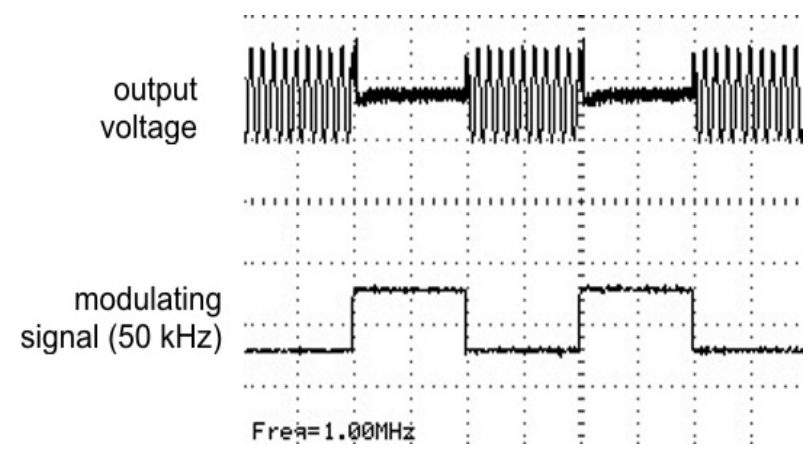

Figure 7. Step response in circuit for a rate of $50 \mathrm{kHz}$. sensor was interrogated (region indicated by the yellow square in Figure 10).

\subsection{Data processing}

Since the resistance $R$ is directly proportional to the conductivity of the material, and the capacitance $C$ is directly proportional to the electric permittivity of the material, from equations (3) and (4) it is possible to show that the amplitudes of the voltage measured in each plateau is proportional to the electrical properties of the fluid at the sensing elements of a planar sensor. For simplicity we will define $V_{x}$ as the voltage of low-frequency component and $V_{\varepsilon}$, as the voltage of high-frequency component. The measured output voltages $V$ * correspond to the conductivity value $x$ at the crossing points according to

$V_{x}=K \cdot \kappa$,

where $K$ is a proportionality factor which depends on electronic circuit and sensor constants. In this way, the individual substance present over a sensing point can be identified by evaluating the output voltage $V_{x}$ and thus an electrically conducting and a non-conducting fluid (e.g. air and water) can be distinguished. In order to discriminate two non-conducting fluids like air and oil, the capacitance or the permittivity of a sensing element must be evaluated. The voltage $V_{\varepsilon}$ is proportional to the relative permittivity $\varepsilon_{\mathrm{r}}$ of the fluid present at a crossing point according to

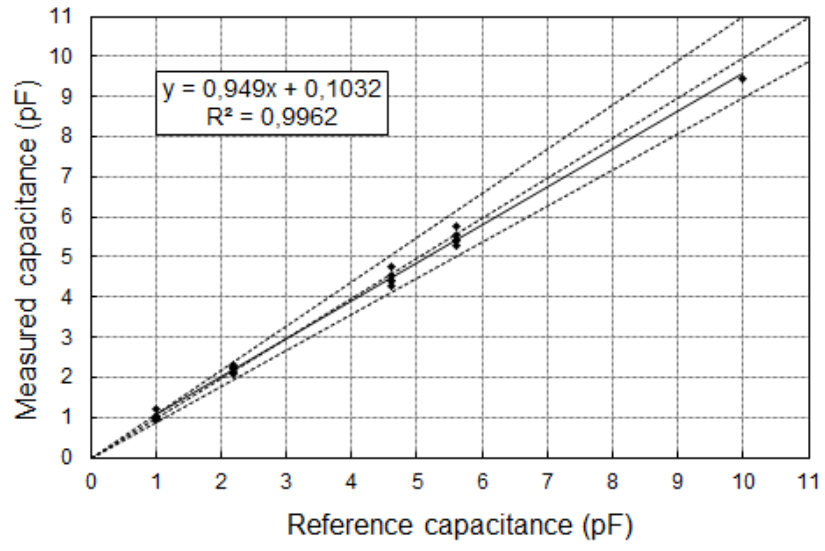

Figure 8 . Parity plot of measured and reference values of capacitance. The dotted lines show the $+/-10 \%$ deviation from ideal line.

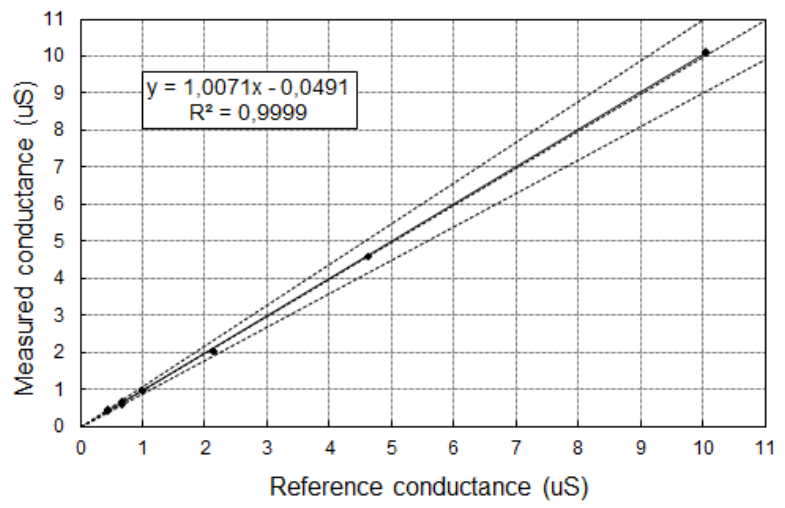

Figure 9. Parity plot of measured and reference values of conductance. The dotted lines show the $+/-10 \%$ deviation from ideal line. 


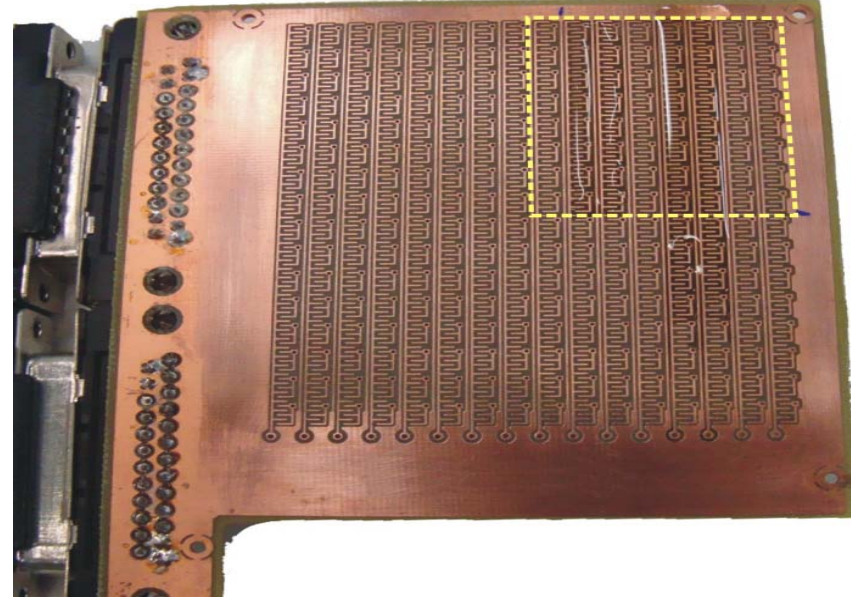

Figure 10. Photography of planar array sensor employed for substance distribution measurement. Region in the sensor indicated by the dotted yellow square is the interrogating area ( $8 \times 8$ single sensors).

\section{Water Oil}

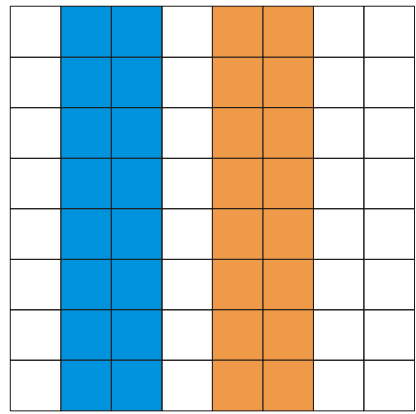

Figure 11. Distribution of substances imposed over the surface of planar sensor.
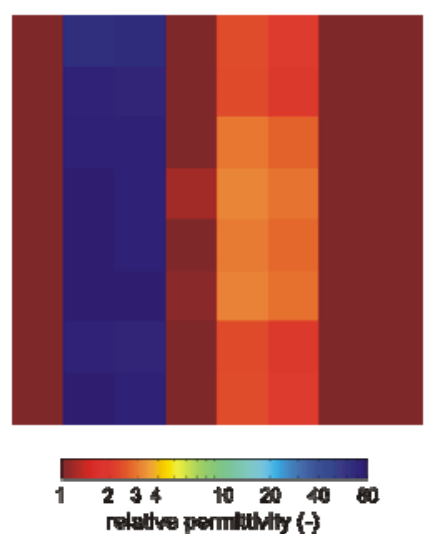
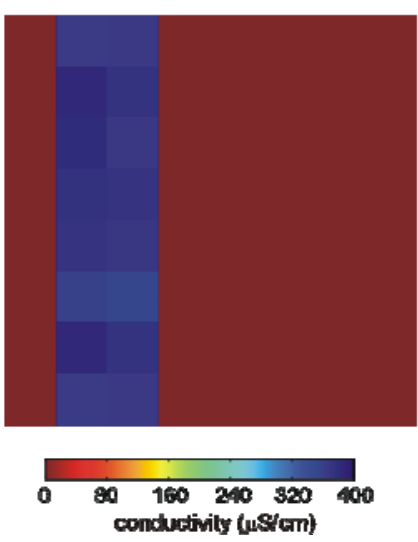

Figure 12. Measured distributions of electrical permittivity and conductivity.

$V_{\varepsilon}=a \cdot \varepsilon_{\mathrm{r}}+b$

where $a$ and $b$ are constants that encompass the specific parameters of the electronics and sensor.

Repeated successive activation of all transmitter electrodes and measurement of currents for all receiver channels gives a three-dimensional data matrix with electrical voltage values denoted by $V(i, j, k)$ which corresponds either to conductivity or permittivity distributions over the pipe's cross section. Here, $i$ and $j$ are the spatial indices (corresponding to the wire numbers
1 to 8 ) and $k$ is the temporal index of each image. Equations (5) and (6) hold for every sensing element in the matrix of a planar sensor. A calibration of all transmitter-receiver pairs is necessary to obtain accurate output readings. Calibration may be performed by measuring the output voltages for two known substances (for instance water and air) and calculating parameters $K(i, j)$ or $a(i, j)$ and $b\left(i_{j}\right)$ for each transmitterreceiver channel.

\subsection{Substance distribution measurement}

In order to show the imaging capability of the spatial distribution of a substance over the sensor surface, a simple experiment was conducted. Tap water and silicone oil were intentionally distributed as two column arrangement over the sensor surface, as illustrated in Figure 11. The electrical conductivity of water, measured with a conductivity meter, was $398 \mu \mathrm{S} / \mathrm{cm}$. The conductivity of oil can be neglected. Reference relative permittivities of air, oil and water are 1, 2.8, and 80, respectively $[19,20]$.

Prior to the start of the experiment a two-point calibration was realized with air and water as references. In this test, 100 frames were acquired and averaged. Figure 12 shows the resulting images, in which a logarithmic colour scale for the permittivity and linear colour scale for conductivity values was used. All three substances involved (air, oil and water) can be clearly recognized in the permittivity image, while in the conductivity one only the conducting from the non-conducting phase is distinguished, as expected. Comparison via visual inspection of the distribution shows good agreement with the measurements.

The measured values of conductivity and permittivity for the sensing elements at row number 4 (middle line of images) were
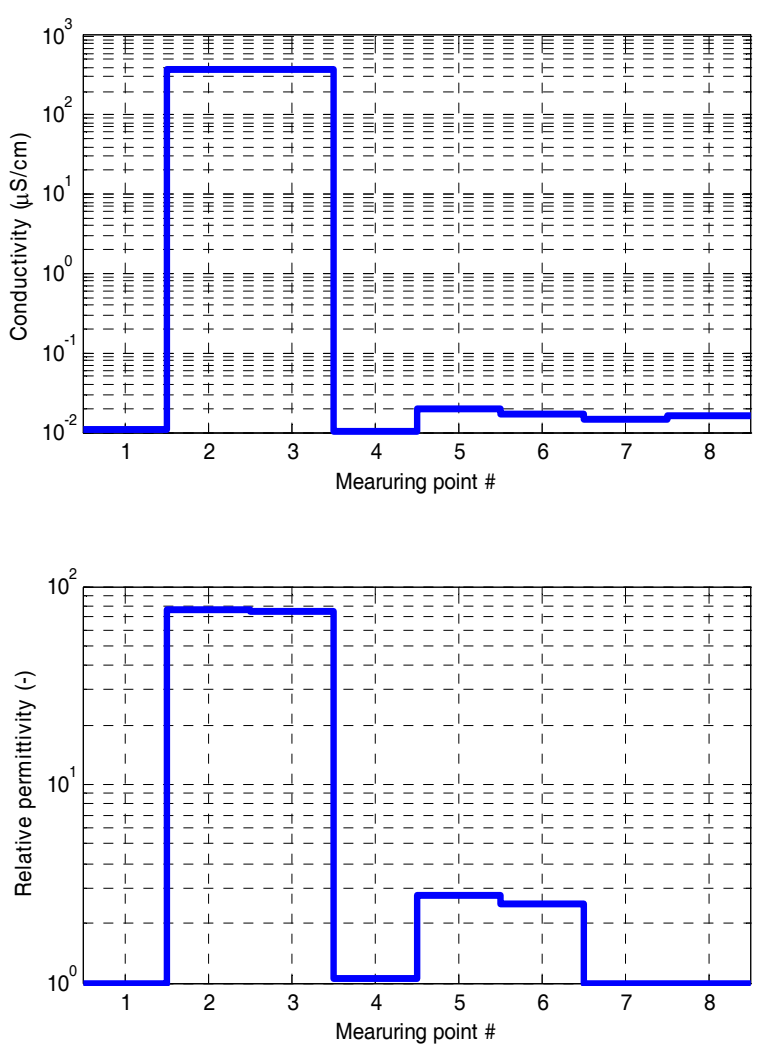

Figure 13. Values of measured electrical conductivity and permittivity taken from sensors at line number 4 (central line of interrogating area). 
taken and plotted in Figure 13, in order to show quantitative data. A logarithmic scale for both permittivity and conductivity is used for comparison purposes. It can be seen that the conductivity values of air and oil are very low (around $10^{-2} \mu \mathrm{S} / \mathrm{cm}$ ) and the conductivity value of water is about $400 \mu \mathrm{S} / \mathrm{cm}$, as expected. Permittivity values for air, oil and water are in good agreement with reference ones. All three substances can be properly distinguished from each other based on their electrical properties.

\section{CONCLUSIONS}

A novel measuring system for multipoint impedance measurement of capacitive and resistive components was presented and tested. The impedance is determined based on the measurement of the amplitudes at two distinct frequencies. The system was evaluated showing appropriated accuracy and fast response. Imaging capability of the multichannel system was explored by operating a planar array sensor. Conductivity and permittivity images are in good agreement with the imposed distribution over sensor surface. Further work will focus on data fusion of the two measured parameter and application to the imaging of dynamic flow.

\section{ACKNOWLEDGEMENT}

Authors acknowledge financial support from PETROBRAS, Brazil and BG Group, Brazil for partially funding this project. E.N. Santos thanks PRH10/ANP/PETROBRAS for doctoral degree scholarship and T.P. Vendruscolo thanks IBP and BG Group, Brazil for a master degree scholarship.

\section{REFERENCES}

[1] R.Pallas-Areny and J.G.Webster, Sensors and Signal Conditioning, 2nd ed., New York: Willey, 2001, ISBN 0471332321.

[2] Agilent, "Agilent Impedance Measurement Handbook", Application Note, Agilent Technologies, 2009. Available at: $<$ http://www.agilent.com>

[3] J.R.Macdonald, W.B.Johnson, "Fundamentals of Impedance Spectroscopy", in: Impedance Spectroscopy, J.R. Macdonald (ed). New York: John Wiley \& Sons, pp. 1-26, 1987, ISBN 0471831220 .

[4] U.Kaatze, Y.Feldman, "Broadband dielectric spectrometry of liquids and biosystems", Measurement Science and Technology, 17, 2006, pp.R17-R35.

[5] H.McCann, D.M.Scott, Process Imaging For Automatic Control Boca Raton, FL: CRC Press., 2005. ISBN 0824759206.

[6] M.J.Da Silva, E.Schleicher, U.Hampel, "Capacitance wire-mesh sensor for fast measurement of phase fraction distributions". Measurement Science and Technology 18, 2007, pp. 2245-2251.
[7] H.M.Prasser, A.Böttger, J.Zschau. "A new electrode-mesh tomograph for gas-liquid flows". Flow Measurement and Instrumentation 9, 1998, pp.111-199.

[8] T.Hermes, M.Bühner, S.Bücher, C.Sundermeier, C.Dumschat, M.Borchardt, K.Cammann, M.Knoll. "An amperometric microsensor array with 1024 individually addressable elements for two-dimensional concentration mapping", Sensors and Actuators B: Chemical, 21, 1994, pp.33-37.

[9] R.S.Saxena, R.K.Bhan, A.Aggrawal. "A new discrete circuit for readout of resistive sensor arrays". Sensors and Actuators A: Physical, 149, 2009, pp.93-99.

[10] A.V.Mamishev, K.Sundara-Rajan, F.Yang, Y.Du, M.Zahn, "Interdigital sensors and transducers", Proceedings of the IEEE, 92, 2004, pp.808-845.

[11] M.J.Da Silva, E.Schleicher, U.Hampel. "Advanced wire-mesh sensor technology for fast flow imaging", in: IEEE International Workshop on Imaging Systems and Techniques 2009, Shenzhen. Proceedings of IST 2009, pp. 253-257.

[12] M.J.Da Silva, T.Sühnel, E.Schleicher, R.Vaibar, D.Lucas, U.Hampel. "Planar array sensor for high-speed component distribution imaging in fluid flow applications”. Sensors 7, 2007, pp. 2430-2445.

[13] S.Thiele, M.J.Da Silva, U.Hampel, "Capacitance Planar Array Sensor for Fast Multiphase Flow Imaging". IEEE Sensors Journal, v. 9, 2009, pp. 533-540.

[14] M.Damsohn, H.M.Prasser "High-speed liquid film sensor with high spatial resolution", Measurement Science and Technology 20, 2009, 114001.

[15] E.Dykesteen, A.Hallanger, E.Hammer, E.Samnoy, R.Thorn, "Non-Intrusive Three-Component Ratio Measurement Using an Impedance Sensor," Journal of Physics E-Scientific Instruments 18,1985 , pp. $540-544$.

[16] F.García-Golding, M.Giallorenzo, N.Moreno, V.Chang, "Sensor for Determining the Water-Content of Oil-In-Water Emulsion by Specific Admittance Measurement," Sensors and Actuators A 47, 1995, pp. 337-341.

[17] M.J.Da Silva, E.Schleicher, U.Hampel, "A novel needle probe based on high-speed complex permittivity measurements for investigation of dynamic fluid flows". IEEE Transactions on Instrumentation and Measurement 56, 2007, pp.1249-1256.

[18] A.Kimoto, T.Kitajima, "An optical, electrical and ultrasonic layered single sensor for ingredient measurement in liquid" Measurement Science and Technology, 21, 2010, 035204.

[19] M.J.Da Silva, Impedance sensors for fast multiphase flow measurement and imaging. TUD Press, Dresden, 2008, ISBN 978-3940046994.

[20] D.R.Lide, CRC Handbook of Chemistry and Physics, 85th ed., Boca Raton, FL: CRC Press, 2005, pp. 6-155-6-177, ISBN 978-0849304859. 